\title{
Mantenimento del controllo di malattia nei pazienti con acromegalia convertiti da terapia con analoghi del recettore della somatostatina iniettabili all'octreotide orale
}

\author{
Giulia Le Grazie $^{1} \cdot$ Luigi Laviola $^{1}$
}

Accettato: 1 dicembre 2020 / Pubblicato online: 9 aprile 2021

(c) The Author(s) 2021

Commento a:

Maintenance of acromegaly control in patients switching from injectable somatostatin receptor ligands to oral octreotide.

S.L. Samson, L.B. Nachtigall, M. Fleseriu, M.B. Gordon, M. Bolanowski, A. Labadzhyan, E. Ur, M. Molitch, W.L. Ludlam, G. Patou, A. Haviv, N. Biermasz, A. Giustina, P.J. Trainer, C.J. Strasburger, L. Kennedy, S. Melmed.

J Clin Endocrinol Metab (2020) 105(10):e3785-e3797

Gli analoghi del recettore della somatostatina rappresentano i farmaci di prima linea nel trattamento dell' acromegalia. Solitamente vengono impiegati in pazienti con persistenza di malattia dopo la chirurgia, ma è stato dimostrato come la loro assunzione nel periodo preoperatorio possa favorire un maggior controllo biochimico a tre mesi dall'intervento. Infine, sono considerati nei soggetti le cui comorbidità escludano tale opzione terapeutica o che rifiutino la chirurgia [1].

Le formulazioni disponibili al momento sono somministrate per via iniettiva con cadenza mensile: octreotide e pasireotide (quest'ultimo al momento non indicato in prima linea) tramite iniezione intramuscolare, lanreotide in sede sottocutanea profonda. La terapia iniettiva può condizionare negativamente la qualità della vita dei pazienti che spesso vivono sentimenti come ansia e frustrazione [2], legati anche alla perdita di indipendenza che tale modalità di somministrazione spesso comporta.

$\triangle$ L. Laviola

luigi.laviola@uniba.it

1 Sezione di Medicina Interna, Endocrinologia, Andrologia e Malattie Metaboliche, Dipartimento dell'Emergenza e dei Trapianti di Organi, Università degli Studi di Bari "Aldo Moro", Bari, Italia
Da alcuni anni vengono condotti studi per testare una formulazione orale dell'octreotide. Fra questi, uno di fase III ha dimostrato che il $65 \%$ dei pazienti con buona risposta agli agonisti iniettivi mantiene il controllo biochimico e la riduzione dei sintomi con il passaggio alla formulazione orale [3].

Il CHIASMA OPTIMAL (Octreotide capsules vs Placebo Treatment in MultinationAL centers) è uno studio prospettico, multicentrico, randomizzato, doppio-cieco, condotto su pazienti con comprovata attività di malattia e con controllo biochimico (IGF-1 $\leq 1,0 \times \mathrm{ULN}$ ) raggiunto con octreotide o lanreotide iniettivi.

I soggetti arruolati sono stati assegnati al gruppo trattato con octreotide $20 \mathrm{mg}$, con un'assunzione da due a quattro capsule al giorno, o al gruppo a destinato a placebo.

A 36 settimane, il controllo di malattia valutato con IGF-1 $(\leq 1,0 \times$ ULN) è stato mantenuto nel $58,2 \%$ dei pazienti in trattamento con octreotide contro il $19,4 \%$ con placebo $(p=0,008)$. Discorso analogo per quanto riguarda il valore target del $\mathrm{GH}(<2,5 \mathrm{ng} / \mathrm{ml})$ confermato nel $77,7 \%$ dei soggetti con octreotide e nel $30,4 \%$ con placebo $(p=0,0007)$.

Inoltre, i pazienti che hanno ricevuto il placebo hanno presentato un tempo medio di perdita della risposta (IGF1 $>1,0$ o $\geq 1,3 \times \mathrm{ULN}$ ) pari a 16 settimane, quadro che non si è creato durante le 36 settimane di studio dai soggetti in terapia con octreotide ( $p<0,0001)$.

Gli effetti avversi sviluppati nel braccio in trattamento sono analoghi a quelli dei farmaci iniettivi, prevalentemente gastrointestinali o alterazioni del metabolismo glucidico, mentre nel braccio trattato con placebo si sono presentati sintomi riconducibili alla riacutizzazione della patologia, quali artralgia, iperidrosi e astenia.

La formulazione orale di octreotide rappresenta un'interessante opzione terapeutica per il trattamento dell'acrome- 
galia, in quanto ridurrebbe gli effetti irritativi e locali legati alle iniezioni, eviterebbe la ricorrenza dei sintomi di malattia al termine del ciclo iniettivo e migliorerebbe la qualità di vita dei pazienti.

Sono necessari ulteriori studi in cui valutare tale opzione terapeutica in pazienti con nuova diagnosi, per testarne l'efficacia in soggetti che non hanno mai assunto farmaci specifici per la patologia.

Funding Note Open access funding provided by Università degli Studi di Torino within the CRUI-CARE Agreement.

Conflitto di interesse L'autrice Giulia Le Grazie dichiara di non avere conflitti di interesse. L'autore Luigi Laviola dichiara di aver ricevuto negli ultimi due anni compensi o finanziamenti dalle seguenti aziende farmaceutiche e/o diagnostiche: Abbott, Boehringer Ingelheim, Astra Zeneca, Lilly Italia, Medtronic, MOVI, Novo Nordisk, Roche, Sanofi Aventis, Takeda (collaborazione scientifica); Abbott, Boehringer Ingelheim, Astra Zeneca, Lilly, Medtronic, Menarini, Movi, MSD, Mundipharma, Novo Nordisk, Roche, Sanofi-Aventis, Takeda (relazioni a convegni, supporto per partecipazione a congressi).

Nota della casa editrice Springer Nature rimane neutrale in riguardo alle rivendicazioni giurisdizionali nelle mappe pubblicate e nelle affiliazioni istituzionali.

Open Access This article is licensed under a Creative Commons Attribution 4.0 International License, which permits use, sharing, adap- tation, distribution and reproduction in any medium or format, as long as you give appropriate credit to the original author(s) and the source, provide a link to the Creative Commons licence, and indicate if changes were made. The images or other third party material in this article are included in the article's Creative Commons licence, unless indicated otherwise in a credit line to the material. If material is not included in the article's Creative Commons licence and your intended use is not permitted by statutory regulation or exceeds the permitted use, you will need to obtain permission directly from the copyright holder. To view a copy of this licence, visit http://creativecommons.org/licenses/by/4.0/.

\section{Bibliografia}

1. Giustina A, Barkhoudarian G, Beckers A et al (2020) Multidisciplinary management of acromegaly: a consensus. Rev Endocr Metab Disord 21(4):667-678

2. Fleseriu M, Fogelfeld L, Gordon MB et al (2020) An evaluation of the Acromegaly Treatment Satisfaction Questionnaire (AcroTSQ) in adult patients with acromegaly, including correlations with other patient-reported outcome measures: data from two large multicenter international studies. Pituitary 23(4):347-358

3. Melmed S, Popovic V, Bidlingmaier M et al (2015) Safety and efficacy of oral octreotide in acromegaly: results of a multicenter phase III trial. J Clin Endocrinol Metab 100(4):1699-1708 